\title{
AVALIAÇÃO DOS RESULTADOS DA METODOLOGIA DE MEDICAMENTOS GATILHO PARA BUSCA DE REAÇÕES ADVERSAS
}

\author{
EVALUATION OF THE RESULTS OF TRIGGER DRUGS METHODOLOGY TO SEARCH ADVERSE REACTIONS
}

Paulo Monteiro Araújo ${ }^{1}$, Carina da Costa Braúna ${ }^{2}$, Veridiana Rebelo dos Santos ${ }^{3}$, Lorena Citó Lopes Resende Santana ${ }^{4}$, Maria do Socorro Rego de Amorim ${ }^{5}$, Mayara Ladeira Coêlho 6

\footnotetext{
${ }^{1}$ Farmacêutico - Mestrando em Ciências Farmacêuticas; Especialista em Farmacologia Clínica e Atenção Farmacêutica; Teresina - Piauí - Brasil. E-mail: paulomonteiroaraujo@hotmail.com.br

${ }^{2}$ Farmacêutica - Mestranda em Ciências Farmacêuticas - UFPI; Teresina - Piauí - Brasil. E-mail: carinabrauna@hotmail.com

${ }^{3}$ Farmacêutica; Teresina - Piauí - Brasil. E-mail: veridianarebelo@gmail.com

${ }^{4}$ Farmacêutica - Hospital Universitário - UFPI; Mestre em Farmacologia dos Produtos Naturais - UFPI; Doutora em Biotecnologia (RENORBIO-UFPI); Teresina - Piauí - Brasil. E-mail: Iorenacito@gmail.com

${ }^{5}$ Enfermeira - Hospital Universitário - UFPI; Especialista em Qualidade e Segurança do Paciente, Terapia Intensiva e Processos Educacionais em Saúde com ênfase em Aprendizagem Significativa; Teresina - Piauí - Brasil. E-mail: helprego@hotmail.com

${ }^{6}$ Farmacêutica - Hospital Universitário - UFPI; Mestre em Ciências Farmacêuticas - UFPI; Doutora em Biotecnologia (RENORBIO-UFPI); Teresina - Piauí - Brasil. E-mail: mayaralcoelho@hotmail.com
}

\section{ABSTRACT}

OBJECTIVE: Understand the results obtained with the implementation of the search for adverse reactions through the Trigger tool methodology and consolidate the types of adverse reactions, the drugs most involved in them and possible points of improvement in the implementation of the search. METHODOLOGY: It is a crosssectional and exploratory study with retrospective data collection. The study period is from March 2017 through February 2018 and included all patients seen using any of the medications listed in the trigger tool list. RESULTS: Was analyzed 6068 detection events in 2726 medical records, of which, in 22 of them, ADRs occurred. The study population was characterized as middle-aged adults, with mean age of 47 years (SD \pm 21 years). The population is equally divided in to gender, with 11 individuals for each sex. Most of the reactions was triggered by antimicrobials or analgesics, and the intravenous route was predominant for the administration of these drugs; these reactions was mostly characterized as B - Bizarre type, with probable causality and mild severity. CONCLUSION: The following improvements were suggested: promotion of the rational use on antimicrobials and analgesics, the use of causality algorithms that involve more comprehensive and in line variables with the adverse reactions investigated.

KEYWORDS: Patient Harm; Patient Safety; Drug-related side effects and adverse reactions. 


\section{RESUMO}

OBJETIVO: Compreender os resultados obtidos com a implementação da busca de reações adversas através da metodologia de medicamentos gatilho (Trigger tool) e consolidar os tipos de reações adversas, os medicamentos mais envolvidos nelas e possíveis pontos de melhoria na implementação da busca. METODOLOGIA: Trata-se de um estudo transversal e exploratório com coleta de dados retrospectiva. O período de estudo fora de março de 2017 até fevereiro de 2018, foram incluídos todos os pacientes atendidos que utilizavam algum dos medicamentos da lista de medicamentos gatilho. RESULTADOS: Foram analisados 6068 eventos de deteç̧ão em 2726 prontuários, do qual, em 22 deles ocorreram RAMs. A população do estudo foi caracterizada como adultos de meia idade, com média de 47 anos ( $D P \pm 21$ anos). Sendo a população igualmente dividida quanto ao gênero, com 11 indivíduos para cada sexo. A maioria das reações foram desencadeadas por antimicrobianos ou analgésicos, e a via endovenosa fora predominante para a administração desses medicamentos, essas reações foram caracterizadas em sua maioria como do tipo B - Bizarras, com causalidade provável e gravidade leve. CONCLUSÃO: As seguintes melhorias foram sugeridas: promoção do uso racional de antimicrobianos e analgésicos, utilização de algoritmos de causalidade que envolvam o uso de variáveis mais abrangentes e alinhadas com as reações adversas ao qual se investiga.

DESCRITORES: Dano ao paciente. Segurança do paciente. Efeitos Colaterais e Reações Adversas Relacionados a Medicamentos.

\section{Como citar este artigo:}

Araújo PM, Braúna CC, Santos VR, Santana LCLR, Amorim MSR, Coêlho ML. Avaliação dos resultados da metodologia de medicamentos gatilho para busca de reações adversas. J. Ciênc. Saúde [internet]. 2018 [acesso em: dia mês abreviado ano];1(3):29-37 . Disponível em: DOI: https://doi.org/10.26694/2595$\underline{0290.20181329-378493}$ 


\section{INTRODUÇÃO}

Os medicamentos são importantes insumos para a assistência à saúde. Seu uso deve sempre objetivar por um efeito benéfico ao indivíduo, com efetividade adequada e toxidade aceitável. No entanto, o dano causado ao paciente por medicamentos possui um custo significativo ao sistema de saúde, mundialmente, este agravo leva a hospitalizações ou ao aumento do tempo de internação, além de maiores índices de morbidade e mortalidade ${ }^{(1)}$.

Desde 2004, a Organização Mundial de Saúde (OMS) em parceria com a aliança mundial para segurança do paciente, cria metas com o objetivo de melhorar a assistência prestada ao paciente. Uma das mais recentes metas criadas por estas instituições foi a de "medicação sem danos". Estima-se que erros de medicação custam anualmente 42 bilhões de dólares, representando $0,7 \%$ do gasto mundial em saúde ${ }^{(2)}$.

As reações adversas fazem parte dos erros de medicamentos que a OMS tenta combater. No estudo de Leendertse et al. (2011), foi estimado o custo médio de uma reação adversa relacionada a medicamento (RAM) do tipo evitável em mais de 5000 euros, considerando ainda a perca da produtividade do indivíduo, que passa a ausentar-se de sua atividade laboral, tem-se somado mais cerca de 1500 euros de custo a sociedade ${ }^{(3)}$.

A farmacovigilância surge como necessidade para um mundo que usa amplamente a intervenção medicamentosa, essa atividade tem por objetivo monitorar a relação risco/benefício dos medicamentos utilizados no cuidado do paciente ${ }^{(4,5)}$. A notificação voluntária de reações adversas é um método de baixo custo para avaliação da segurança de medicamentos na fase de pós-comercialização. Todavia, em países em sua maioria desenvolvidos, buscam metodologias de monitorização com maior capacidade de detectar $\mathrm{RAMs}^{(4)}$.

Em busca da melhoria na qualidade da assistência prestada ao paciente, diversas organizações de saúde criaram metodologias capazes de detectar e avaliar reações adversas. Um método promissor surgiu dentre essas diversas metodologias, o de medicamentos gatilho (Trigger Tools). Método baseado na revisão retrospectiva de registros médicos ao qual utiliza uma lista de gatilhos, afim de, determinar quais prontuários serão revisados em busca de RAMs $^{(6)}$.

Nessa metodologia eventos específicos como a prescrição de certas drogas, exames com valores anormais, suspensões abruptas de medicamentos, servem como gatilhos para a sondagem dos prontuários. Por exemplo, o uso da naloxona associado aos sinais de intoxicação de opioides, ou o aumento da creatina com certas medicações nefrotóxicas, entre outros $^{(7)}$.

Todavia, a detecção dos danos relacionados a medicamentos é complexa e cheia de desafios, principalmente no tocante da definição e caracterização dos mesmos. A qualidade e disponibilidade da informação e o desenvolvimento de algoritmos com alta exatidão são subsídios necessários para a melhoria da captação de RAMs, principalmente quando se utiliza a metodologia de medicamentos gatilho ${ }^{(8)}$.

Esse trabalho teve por objetivo: Compreender os resultados obtidos com a implementação da busca de RAMs com o uso da metodologia de medicamentos gatilho, consolidar os tipos de RAMs, os medicamentos mais envolvidos nelas, por fim, propor possíveis pontos de melhoria.

\section{METODOLOGIA}

Trata-se de um estudo transversal e exploratório com coleta de dados retrospectiva. Realizado em um hospital universitário de grande porte - com 175 leitos de enfermaria e 15 leitos de UTI - que atende pacientes de média e alta complexidade, com mais de 18 anos de idade completos. O período de estudo foi de março de 2017 até fevereiro de 2018, e fora incluído toda a população que utilizava algum dos medicamentos da lista de gatilhos.

Este trabalho seguiu todos os preceitos éticos exigidos pelo comitê de ética em pesquisa, aprovado com o número 94522018.3 .0000 .8050 . 
As reações adversas foram identificadas utilizando-se uma lista de medicamentos gatilho (Tabela 1) criada na própria instituição. Com ajuda do sistema de prescrição eletrônica triava-se aqueles pacientes que durante algum período da internação recebeu alguma das medicações contidas na lista, posteriormente avaliando seu prontuário em busca de indicações de RAMs.

Tabela 1 - Medicamentos gatilho e os sinais observados na busca de reações adversas a medicamentos através da metodologia de trigger tools.

\begin{tabular}{ll}
\hline Medicamentos gatilho & Sinais observados \\
\hline Hidrocortisona & Reações alérgicas \\
\hline $\begin{array}{l}\text { Dexclorfeniramina } \\
\text { Loratadina } \\
\text { Prometazina }\end{array}$ & Reações alérgicas \\
\hline $\begin{array}{l}\text { Bromoprida } \\
\text { Metoclopramida }\end{array}$ & Vomito e náuseas \\
\hline Naloxona & Miose, bradicardia, \\
& bradipneia e letargia \\
\hline
\end{tabular}

Fonte: Os autores.

As RAMs foram classificadas de acordo com a sua gravidade $(\mathrm{WHO})^{(9)}$, causalidade (Algoritmo de Naranjo) $)^{(10)}$ e tipo (Edwards e Aronson) $)^{(11)}$, utilizou-se os algoritmos e classificações propostos por cada referência. O sinal clínico foi definido por relato no prontuário, seja pela equipe médica ou pela equipe de enfermagem.

A revisão primária foi realizada por estagiários do setor de vigilância em saúde do hospital, posteriormente revisado por uma equipe de residentes de farmácia e por fim pela farmacêutica responsável do projeto.

Para análise e tratamento dos dados utilizou-se o programa Microsoft ${ }^{\circledR}$ Office Excel 2019. Os resultados foram descritos em estática descritiva, utilizou-se a distribuição de frequências (absoluta e porcentagem) e medidas de dispersão (desvio padrão) somente para as idades.

\section{RESULTADOS}

Foram analisados 6068 eventos de detecção em 2726 prontuários, do qual, em 22 deles ocorreram RAMs. A população do estudo foi caracterizada como adultos de meia idade, com média de 47 anos (idade mínima de 19 anos e máxima de 85 anos), apresentando o desvio padrão de 21 anos. Sendo a população igualmente dividida quanto ao gênero, sendo 11 do sexo masculino e 11 do sexo feminino (Tabela 2).

Tabela 2 - Idade, faixa etária e gênero dos pacientes que apresentaram reações adversas detectadas pela metodologia de medicamentos gatilho.

\begin{tabular}{cc}
\hline Idade & \\
\hline Média & 47 \\
Desvio padrão & 21 \\
\hline Faixa etária & $\mathbf{N}(\%)$ \\
\hline $0-19$ & $2(9)$ \\
$20-39$ & $8(36)$ \\
$40-59$ & $5(23)$ \\
$60-89$ & $6(27)$ \\
$>90$ & $1(5)$ \\
\hline Sexo & $\mathbf{N ~ ( \% )}$ \\
\hline Feminino & $11(50)$ \\
Masculino & $11(50)$ \\
\hline
\end{tabular}

Fonte: Os autores.

A tabela 3 destaca que a maior quantidade de reações adversas foi desencadeada por medicamentos administrados pela via endovenosa 20 (91\%). Dentre os medicamentos avaliados, os antimicrobianos e os analgésicos foram as classes medicamentosas que apresentaram a maior incidência de reações adversas relacionadas a eles, 13 (59\%) e 6 (27\%), respectivamente. E ainda é possível destacar que os medicamentos que mais causaram RAMs para a população do estudo foram a Morfina 4 (18\%) e a Piperacilina + Tazobactam 2 (9\%). 
Tabela 3 - Classe terapêutica e vias de administração dos medicamentos desencadeadores das reações adversa.

\begin{tabular}{lc}
\hline Classe terapêutica & N (\%) \\
\hline Antimicrobiano & $13(59)$ \\
Analgésico & $6(27)$ \\
Antiemético & $1(5)$ \\
Antiulceroso & $1(5)$ \\
Quimioterápico & $1(5)$ \\
\hline Via de administração & $\mathbf{N}(\%)$ \\
\hline Parenteral & $20(91)$ \\
Enteral & $2(9)$ \\
\hline
\end{tabular}

Fonte: Os autores.

De acordo com a tabela 4 a classe terapêutica dos gatilhos que identificaram a maior quantidade de reações adversas foram os anti-histamínicos 13 (54\%), corticosteroides sistêmicos 4 (17\%) e os antieméticos 5 (21\%).

Tabela 4 - Medicamentos gatilho seguidos do percentual de identificação das reações adversas relacionadas a medicamentos.

\begin{tabular}{lc}
\hline Medicamento gatilho & N (\%) \\
\hline Dexclorfeniramina & $6(25)$ \\
Metoclopramida & $5(21)$ \\
Hidrocortisona & $4(17)$ \\
Loratadina & $4(17)$ \\
Prometazina & $3(13)$ \\
Naloxona & $2(8)$ \\
\hline Total & $24 *$ \\
\hline
\end{tabular}

*Duas reações adversas apresentaram dois medicamentos gatilho.

Fonte: Os autores.

A tabela 5 mostrou que a gravidade na maioria as reações adversas foram consideradas leve 11 (50\%), o sinal clínico mais observado foi o de quadro alérgico 15 (68\%), duas reações caracterizadas pelo quadro alérgico foram as mais graves, ambas desencadeadas por antimicrobianos. Quanto a classificação das RAMs as de tipo $B$ (Bizarros) foram as mais encontradas, seguida por reações do tipo A (Aumento) e do tipo C (Crônico). De acordo com a causalidade a maioria das reações foi do tipo provável 19 (86\%).
Tabela 5 - Caracterização da RAMs encontradas através da metodologia de trigger tools em um hospital universitário.

\begin{tabular}{lc}
\hline Gravidade da reação & N (\%) \\
\hline Grave & $2(9)$ \\
Moderada & $9(41)$ \\
Leve & $11(50)$ \\
\hline Tipo de sinal observado & $\mathbf{N}(\%)$ \\
\hline Intoxicação & $2(9)$ \\
Náuseas e vômitos & $5(23)$ \\
Reação alérgica & $15(68)$ \\
\hline Classificação da RAM & $\mathbf{N}(\%)$ \\
\hline A - Relacionado à dose (Aumento) & $4(18)$ \\
B - Não relacionado à dose (Bizarro) & $12(55)$ \\
C - Relacionado à dose e ao tempo de & $4(18)$ \\
uso (Crônico) & $1(5)$ \\
D - Relacionado ao tempo de uso & $1(5)$ \\
(Atraso) & $\mathbf{N}(\%)$ \\
F - Falha inesperada de terapia (Falha) & $3(14)$ \\
\hline Causalidade & $19(86)$ \\
\hline Possível & \\
Provável & \\
\hline Fonte: Os autores. & \\
&
\end{tabular}

No estudo de Lobo et al. (2013), realizado em um hospital da região nordeste com porte e características próximas ao hospital universitário desse estudo, concluiu-se que a faixa de idade mais afetada por RAMs foi a adulta, e o gênero não influenciou na incidência das reações adversas ${ }^{(12)}$.

Para Oliveira et al. (2018), que avaliou a suspeita de reações adversas, a população adulta também foi a mais afetada, todavia os indivíduos do sexo feminino nessa amostragem foram significativamente mais afetados pelas RAMs, parcialmente essa diferença pode ser justificada por diferentes perfis de atendimento das instituições, na instituição de Oliveira, há atendimento de referência a gestantes de alto risco ${ }^{(13)}$. 
A administração pela via parenteral 20 (91\%) foi a mais utilizada para os medicamentos causadores das RAMs, com destaque de que todos foram por via endovenosa. Esta via apresenta maior risco na segurança da administração e no desenvolvimento de complicações durante o tratamento, hoje seu uso tenta ser racionalizado, promove-se a troca por terapias orais, assim que possível, principalmente quando trata-se de antimicrobianos $^{(14)}$.

Antimicrobianos foram os principais desencadeadores de RAMs para a realidade do estudo. No estudo de Tamma e colaboradores (2017), foi observado que $20 \%$ dos tratamentos com antimicrobianos com finalidade curativa apresentaram algum tipo de RAM. Além disso, observou-se que um quinto dos tratamentos com antimicrobianos nesta instituição poderia ter sido evitado, pois não tinham indicação clínica para seu uso(15). Esse passa a ser um ponto interessante de intervenção do farmacêutico clínico, atuar na promoção do uso racional de antimicrobianos ${ }^{(16)}$.

Opioides foram os segundos maiores causadores de RAMs e são amplamente utilizados para o tratamento de dores de intensidade média ou maior. O risco de morte cresce proporcionalmente ao aumento da dose, principalmente quando ultrapassa o equivalente a 50 mg de morfina em 24 horas. É notável a maior capacidade dessa classe medicamentosa de desencadear reações alérgicas em comparação a analgésicos não opioides. Todavia apesar das reações de origem alérgica serem em sua maioria inevitáveis, as de intoxicação podem ser prevenidas com o acompanhamento mais a fundo do perfil dose/resposta de cada paciente. Passo possível pela implementação de um sistema efetivo de acompanhamento clínico ${ }^{(17)}$.

A Piperacilina associada ao Tazobactam foi o medicamento com maior quantidade de reações adversas relatadas. Em acordo com o estudo de Keller e colaboradores (2018), pois apesar desse medicamento possuir uma das menores incidências de RAMs, seu uso em uma ampla variedade de tratamentos, contribui para o alto valor absoluto de reações para esse medicamento $^{(18)}$.
A grande quantidade de reações do tipo B é preocupante, pois em sua maioria, esse tipo está relacionado a reações de hipersensibilidade, imunológicas, idiossincráticas ou de mecanismos desconhecidos. Esse tipo de reação vem de forma inesperada e súbita, o que leva a uma alta taxa de mortalidade frente aos outros tipos de reação(11).

As reações de tipo B podem desencadear cascatas moduladas por linfócitos ou por imunoglobulinas $E$ (IgE), ao qual, quando ativadas de forma exagerada, podem levar a taquicardia, hipotensão e até mesmo em casos mais graves parada cardiorrespiratória. Esse tipo de condição clínica, necessita do uso de corticosteroides sistêmicos e anti-histamínicos, afim de modular a reação alérgica, e controlar os sintomas cutâneos e circulatórios ${ }^{(19)}$.

Em sua maioria, as RAMs encontradas nesse estudo foram classificadas como prováveis 19 (86\%). O estudo de Laatikainen e colaboradores (2016) confrontou os resultados obtidos pelo algoritmo de Naranjo, frente a uma metodologia desenvolvida pela própria instituição, a qual utilizava perguntas definidas, todavia com flexibilidade para confrontação teórica (mecanismo farmacológico) e laboratorial da RAM. Fora obtido um melhor nível de determinação de causalidade, o mesmo ainda sugere que um menor período de tempo da revisão dos dados, pode vir a melhorar a determinação de causalidade ${ }^{(20)}$.

Varallo et al. (2017), sugeriu que um melhor manejo na determinação dos triggers e a avaliação de acordo com um julgamento clínico por uma equipe qualificada, permitiram a determinação de reações com causalidade definida, em seu estudo(21).

Classificação que se mostra praticamente impossível no algoritmo de Naranjo pelas perguntas 4, 5 e 6, as quais praticamente tem suas respostas imutáveis pela prática clínica atual. Já que, raramente o paciente e reexposto ao possível causador da RAM, as bulas de diversos medicamentos relatam parcialmente as mesmas reações adversas e por raramente existir a administração de medicamentos placebo ${ }^{(20)}$. 


\section{CONCLUSÃO}

As reações adversas identificadas através desta metodologia foram em sua maioria reações alérgicas, do tipo B - Bizarro e de causalidade provável. Com destaque para os antimicrobianos e os analgésicos, que foram os maiores desencadeadores de RAMs. Os medicamentos gatilho mais encontrados foram os antihistamínicos, corticosteroides sistêmicos e os antieméticos.

Quanto as melhorias, sugere-se o maior envolvimento do farmacêutico no cuidado ao paciente, a fim de evitar o uso de antimicrobianos sem indicação correta e a melhor otimização da dose no uso dos analgésicos. Além disso, o algoritmo de Naranjo, apesar de amplamente utilizado, mostra sua defasagem frente a realidade atual da assistência à saúde. É recomendado assim, o uso de algoritmos de causalidade, que envolvam o uso de variáveis mais abrangentes e alinhadas aos tipos de medicamentos com que se trabalha.

\section{REFERÊNCIAS}

1. Falconer N, Barras M, Martin J, Cottrell N. Defining and classifying terminology for medication harm: a call for consensus. Eur J Clin Pharmacol [Internet]. 11 de Fevereiro de 2019;75(2):137-45. Disponível em:

http://www.ncbi.nlm.nih.gov/pubmed/30310967

2. Donaldson LJ, Kelley ET, Dhingra-Kumar N, Kieny M-P, Sheikh A. Medication Without Harm: WHO's Third Global Patient Safety Challenge. Lancet [Internet]. 29 de Abril de 2017;389(10080):1680-1. Disponível em:

http://www.ncbi.nlm.nih.gov/pubmed/28463129

3. Leendertse AJ, Van Den Bemt PMLA, Poolman JB, Stoker LJ, Egberts ACG, Postma MJ. Preventable hospital admissions related to medication (HARM):

Cost analysis of the HARM study. Value Heal [Internet].
Janeiro de 2011;14(1):34-40. Disponível em: http://www.ncbi.nlm.nih.gov/pubmed/21211484

4. Botelho SF, Reis AMM. Planos de minimização de riscos em farmacovigilância: uma ação de saúde pública para promoção da segurança de medicamentos. Cien Saude Colet [Internet]. Dezembro de 2015;20(12):3897-905. Disponível em:

http://www.scielo.br/scielo.php?script=sci arttext\&pi $\mathrm{d}=$ S1413-81232015001203897\&lng=pt\&tlng=pt

5. Wise L, Parkinson J, Raine J, Breckenridge A. New approaches to drug safety: a pharmacovigilance tool kit. Nat Rev Drug Discov [Internet]. 18 de Outubro de 2009;8(10):779-82. Disponível em:

http://www.nature.com/articles/nrd3002

6. Musy SN, Ausserhofer D, Schwendimann R, Rothen HU, Jeitziner M-M, Rutjes AW, et al. Trigger Tool-Based Automated Adverse Event Detection in Electronic Health Records: Systematic Review. J Med Internet Res [Internet]. 30 de Maio de

2018;20(5):e198. Disponível em:

http://www.jmir.org/2018/5/e198/

7. Resar RK, Rozich JD, Classen D. Methodology and rationale for the measurement of harm with trigger tools. Qual Saf Health Care [Internet]. 1 de Dezembro de 2003;12 Suppl 2(90002):ii39-45.

Disponível em:

http://qualitysafety.bmj.com/lookup/doi/10.1136/qhc. $\underline{12 . s u p p l ~ 2 . i i 39}$

8. Walter SR, Gallego B. Detecting Adverse Drug Events: Accuracy and Generalizability. Ther Innov Regul Sci [Internet]. 30 de Dezembro de 2018; Disponível em: http://journals.sagepub.com/doi/10.1177/2168479018 $\underline{820045}$

9. World Health Organization. The Importance of Pharmacovigilance - Safety Monitoring of medicinal products [Internet]. 2002. Geneva; [citado 3 de Janeiro de 2019]. Disponível em: http://apps.who.int/medicinedocs/en/d/Js4893e/

10. Naranjo CA, Busto U, Sellers EM, Sandor P, Ruiz I, Roberts EA, et al. A method for estimating the probability of adverse drug reactions. Clin Pharmacol Ther [Internet]. Agosto de 1981;30(2):239-45. 
Disponível em:

http://www.ncbi.nlm.nih.gov/pubmed/7249508

11. Edwards IR, Aronson JK. Adverse drug reactions: definitions, diagnosis, and management. Lancet (London, England) [Internet]. 7 de Outubro de 2000;356(9237):1255-9. Disponível em:

http://www.ncbi.nlm.nih.gov/pubmed/11072960

12. Lobo MGA de A, Pinheiro SMB, Castro JGD, Momenté VG, Pranchevicius M-CS. Adverse drug reaction monitoring: support for pharmacovigilance at a tertiary care hospital in Northern Brazil. BMC Pharmacol Toxicol [Internet]. 8 de Janeiro de 2013;14:5. Disponível em:

http://www.ncbi.nlm.nih.gov/pubmed/23298396

13. Oliveira AM de, Rodrigues VAV, Passerini JP, Pedreiro PBZ, Minto BA. Queixas técnicas e reações adversas a medicamentos notificadas em um hospital regional (Brasil): um estudo transversal. ABCS Heal Sci [Internet]. 15 de Maio de 2018;43(1):61-6. Disponível em:

https://www.portalnepas.org.br/abcshs/article/view/1 $\underline{015}$

14. Berrevoets MAH, Pot JLW, Houterman AE, Dofferhoff ASM, Nabuurs-Franssen $\mathrm{MH}$, Fleuren $\mathrm{HWHA}$, et al. An electronic trigger tool to optimise intravenous to oral antibiotic switch: a controlled, interrupted time series study. Antimicrob Resist Infect Control [Internet]. 15 de Dezembro de 2017;6(1):81. Disponível em:

http://www.ncbi.nlm.nih.gov/pubmed/28824799

15. Tamma PD, Avdic E, Li DX, Dzintars K, Cosgrove SE. Association of Adverse Events With Antibiotic Use in Hospitalized Patients. JAMA Intern Med [Internet]. 1 de Setembro de 2017;177(9):1308-15. Disponível em: http://www.ncbi.nlm.nih.gov/pubmed/28604925

16. Santana KDS, Horácio BO, Silva JE, Cardoso Júnior CDA, Geron VLMG, Terra Júnior AT. O papel do profissional farmacêutico na promoção da saúde e do uso racional de medicamentos. Rev Científica FAEMA [Internet]. 12 de Abril de 2018;9(1):399. Disponível em: http://www.faema.edu.br/revistas/index.php/RevistaFAEMA/article/view/538
17. Genco EK, Forster JE, Flaten H, Goss F, Heard KJ, Hoppe J, et al. Clinically Inconsequential Alerts: The Characteristics of Opioid Drug Alerts and Their Utility in Preventing Adverse Drug Events in the Emergency Department. Ann Emerg Med [Internet]. Fevereiro de 2016;67(2):240-248.e3. Disponível em: http://www.ncbi.nlm.nih.gov/pubmed/26553282

18. Keller SC, Williams D, Gavgani M, Hirsch D, Adamovich J, Hohl D, et al. Rates of and Risk Factors for Adverse Drug Events in Outpatient Parenteral Antimicrobial Therapy. Clin Infect Dis [Internet]. 6 de Janeiro de 2018;66(1):11-9. Disponível em: http://www.ncbi.nlm.nih.gov/pubmed/29020202

19. Ring J, Brockow K. Adverse Drug Reactions: Mechanisms and Assessment. Eur Surg Res [Internet]. 2002;34(1-2):170-5. Disponível em: https://www.karger.com/Article/FullText/48905

20. Laatikainen $\mathrm{O}$, Sneck S, Bloigu R, Lahtinen M, Lauri T, Turpeinen M. Hospitalizations Due to Adverse Drug Events in the Elderly-A Retrospective Register Study. Front Pharmacol [Internet]. 5 de Outubro de 2016;7:358. Disponível em:

http://journal.frontiersin.org/article/10.3389/fphar.20 $\underline{16.00358}$

21. Varallo FR, Dagli-Hernandez C, Pagotto C, de Nadai TR, Herdeiro MT, de Carvalho Mastroianni P. Confounding Variables and the Performance of Triggers in Detecting Unreported Adverse Drug Reactions. Clin Ther [Internet]. Abril de 2017;39(4):686-96. Disponível em: http://www.ncbi.nlm.nih.gov/pubmed/27913030 
Sources of funding: No Conflict of interest: No Accepted: 2019/02/06

Publishing: 2019/12/24
Corresponding Address: Paulo Monteiro Araújo.

Teresina, Piauí, Brasil. E-mail:

paulomonteiroaraujo@hotmail.com.br 